\title{
ChemComm
}

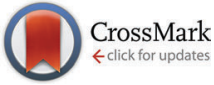

Cite this: Chem. Commun., 2016, 52, 9224

Received 12th May 2016 Accepted 2nd June 2016

DOI: $10.1039 / \mathrm{c} 6 \mathrm{cc} 04011 \mathrm{j}$

www.rsc.org/chemcomm

\section{Paramagnetic lanthanide chelates for multicontrast MRI $\dagger$}

\author{
Nevenka Cakić, ${ }^{a}$ Tanja Savić, ${ }^{a}$ Janice Stricker-Shaver, ${ }^{a}$ Vincent Truffault, ${ }^{b}$ \\ Carlos Platas-Iglesias, ${ }^{c}$ Christian Mirkes, ${ }^{d}$ Rolf Pohmann, ${ }^{d}$ Klaus Scheffler*de and \\ Goran Angelovski*a
}

The preparation of a paramagnetic chelator that serves as a platform for multicontrast MRI, and can be utilized either as a $T_{1}$-weighted, paraCEST or ${ }^{19} \mathrm{~F}$ MRI contrast agent is reported. Its europium(III) complex exhibits an extremely slow water exchange rate which is optimal for the use in CEST MRI. The potential of this platform was demonstrated through a series of MRI studies on tube phantoms and animals.

Visualization of various biological processes that take place at cellular and molecular levels is the main goal of modern diagnostic and molecular imaging techniques. Due to different sensitivity, penetration depth, spatial or temporal resolution properties of the available imaging methods, the development of multimodal imaging experienced great advancements in the last decade. ${ }^{1,2}$ Nevertheless, a combination of two (or more) techniques is often challenging, requiring integration of different physical phenomena in the common hardware, or careful design of multimodal imaging probes. ${ }^{3,4}$

Today, magnetic resonance imaging (MRI) is one of the most powerful imaging tools capable of displaying an excellent soft tissue contrast and furthermore different types of contrasts. A number of diverse MRI contrast agents can improve the specificity of MRI measurements and, based on their nature, can provide different types of information. The most commonly used ${ }^{1} \mathrm{H}$-MRI contrast agents are paramagnetic $\mathrm{Gd}^{3+}$-based complexes or superparamagnetic iron-oxide based nanoparticles $\left(T_{1}\right.$ - and $T_{2}$-shortening

\footnotetext{
${ }^{a}$ MR Neuroimaging Agents, Max Planck Institute for Biological Cybernetics, 72076 Tübingen, Germany. E-mail: goran.angelovski@tuebingen.mpg.de

${ }^{b}$ Max Planck Institute for Developmental Biology, 72076 Tübingen, Germany

${ }^{c}$ Centro de Investigaciones Cientificas Avanzadas (CICA) and Departamento de Quimica Fundamental, Universidade da Coruña, 15008 A Coruña, Spain

${ }^{d}$ High-Field Magnetic Resonance, Max Planck Institute for Biological Cybernetics,

72076 Tübingen, Germany. E-mail: klaus.scheffler@tuebingen.mpg.de

${ }^{e}$ Department for Biomedical Magnetic Resonance, University of Tübingen,

72076 Tübingen, Germany

$\dagger$ Electronic supplementary information (ESI) available: Synthetic procedures, descriptions of CEST, ${ }^{19} \mathrm{~F}$ NMR, NMR diffusion and MRI experiments. See DOI: $10.1039 / \mathrm{c} 6 \mathrm{cc} 04011 \mathrm{j}$
}

agents, respectively). ${ }^{5}$ Recently, an entirely different mechanism for altering an MRI contrast, based on chemical exchange saturation transfer (CEST), has been developed. Contrast agents for CEST imaging usually consist of paramagnetic chelates (paraCEST agents) specifically designed to shift the resonances of exchangeable protons $\left(\mathrm{NH}, \mathrm{OH}\right.$ or bound water) further away from the bulk water. ${ }^{6}$ One of the great advantages of CEST is the detectability of its effect in combination with the presaturation RF pulse; only when this RF is applied at the specific frequency of the exchangeable protons, the MRI contrast will appear. Consequently, this method allows the multi-frequency readout and adjustment of the frequencies (e.g. by choice of the group with exchangeable protons or choice of the paramagnetic ion), which can be used for separate detection and visualization of different cellular and tissue environments. ${ }^{7,8}$ Finally, MRI can be performed on heteronuclear and hyperpolarized systems. ${ }^{9}$ Here, the best choice is ${ }^{19} \mathrm{~F}$ NMR because of its high sensitivity, the easy re-tuning of standard MRI instruments from ${ }^{1} \mathrm{H}$ to ${ }^{19} \mathrm{~F}$ nuclei, the high natural abundance of the ${ }^{19} \mathrm{~F}$ isotope and the absence of background signals from intrinsic biomolecules, thus allowing quantitative studies. ${ }^{10,11}$

With such potential to provide diverse information by employing different frequencies and contrast mechanisms, MRI could be exploited for concurrent studies by means of $T_{1}$-weighted, CEST and ${ }^{19} \mathrm{~F}$ imaging protocols. To this aim, it is highly desirable to develop multicontrast agents, probes capable of making use of these different types of MR contrast, thus resulting in a set of unique information related to the studied tissue of interest.

We therefore aimed to prepare an agent that is capable of chelating the paramagnetic ion, possesses frequency-shifted protons in slow exchange with bulk water, and finally bears a sufficient number of fluorine atoms to provide a sizeable ${ }^{19} \mathrm{~F}$ NMR signal (Fig. 1). We designed ligand L, a derivative of DOTA-tetraglycineamide (DOTAM-Gly), ${ }^{12}$ which displays CEST properties and ensures good solubility in aqueous solutions, especially after incorporation of fluorine atoms. The fluorinated moieties were introduced in the molecule at trans positions of the macrocyclic ring through two $\mathrm{CF}_{3}$ groups by using a 4 -(trifluoromethyl)-Lphenylalanine $\left(p-\mathrm{CF}_{3}-\mathrm{Phe}\right)$ derivative and a convenient six-step 


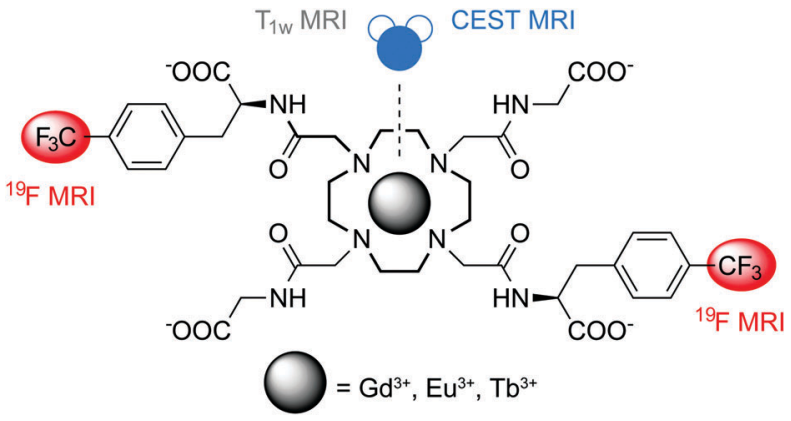

Fig. 1 The chemical structure of chelator $\mathbf{L}$ described in this work: paramagnetic ion makes it suitable for $T_{1}$-weighted or paraCEST MRI, while nearby $\mathrm{CF}_{3}$ groups enable ${ }^{19} \mathrm{~F} N \mathrm{MR}$, concurrently taking advantage of the paramagnetic enhancement effect.

synthetic procedure (ESI $\dagger$ ). The resulting molecule $\mathbf{L}$ underwent complex formation with the $\mathrm{Gd}^{3+}, \mathrm{Eu}^{3+}$ and $\mathrm{Tb}^{3+}$ ions and the properties of GdL, EuL and TbL were further investigated (ESI $\dagger$ ). Their overall charge should be advantageous (negative) due to the presence of four carboxylate groups, while appropriate complex stability necessary for in vivo experiments is ensured by the kinetic inertness provided by the tetraamide moieties. ${ }^{13}$ Finally, rigid benzyl moieties may establish, compared to e.g. aliphatic spacers, a well-defined distance of ${ }^{19} \mathrm{~F}$ nuclei from the paramagnetic center, thus providing advantageous relaxation properties at the frequency of these nuclei. ${ }^{14}$

The longitudinal relaxivity of GdL was calculated by obtaining $T_{1}$ values of a series of buffered solutions at different complex concentrations. The resulting value of $3.1 \mathrm{mM}^{-1} \mathrm{~s}^{-1}$ is expected for monohydrated tetraamide complexes with a slow water exchange rate. ${ }^{15}$ CEST measurements were performed by selective presaturation of the EuL and TbL samples in incremental steps over a range of frequencies followed by plotting the remaining steady-state bulk water signal, $M_{\mathrm{Z}} / M_{0}$, as a function of saturation frequency (Fig. 2). The resulting Z-spectra of EuL exhibited a strong CEST effect $\left(\sim 50 \%\right.$ at $\left.25{ }^{\circ} \mathrm{C}, 15 \mathrm{mM}, B_{1}=25.0 \mu \mathrm{T}\right)$ centred at $50 \mathrm{ppm}$ relative to bulk water, which is commonly associated with the water molecule directly coordinated to $\mathrm{Eu}^{3+}$. The CEST effect remains strong at $37{ }^{\circ} \mathrm{C}$ along with slight broadening and shifting of the CEST signal (5 ppm) towards the bulk water resonance due to the hyperfine shift effect of $\mathrm{Eu}^{3+}$ (Fig. 2, top). ${ }^{16}$

Water exchange rates at both temperatures were determined using a concentration-independent method previously developed by Sherry and colleagues, ${ }^{16}$ and were confirmed by quantification of the exchange rates as a function of saturation time or saturation power (QUEST and QUESP experiments, respectively). ${ }^{17}$ The values obtained from these experiments revealed extremely slow exchange rates of around 1 and $2 \mathrm{kHz}$ or bound-water lifetimes $\left(\tau_{\mathrm{M}}\right)$ of around $1 \mathrm{~ms}$ and $500 \mu \mathrm{s}$ at 25 and $37^{\circ} \mathrm{C}$, respectively (Table 1). These residence times are as long as those reported very recently for the phosphonate esters of DOTAM-Gly at $25{ }^{\circ} \mathrm{C},{ }^{18}$ likely due to the presence of hydrophobic $p-\mathrm{CF}_{3}-\mathrm{Phe}$ moieties. Consequently, EuL displays an almost optimal $\tau_{\mathbf{M}}$ which lies in the range of $10^{-4}-10^{-2} \mathrm{~s}$, allowing CEST experiments using weaker $B_{1}$ fields. On the other hand, TbL displayed a weak CEST effect for both the inner-sphere water molecule and amide
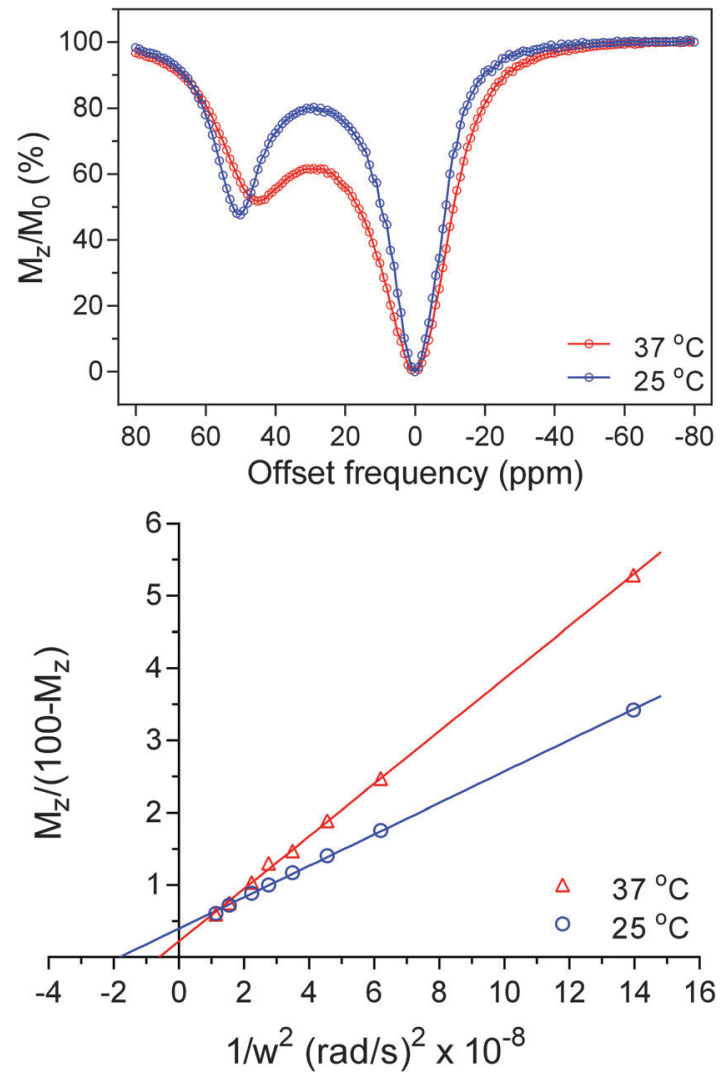

Fig. 2 CEST experiments with EuL (15 mM). (top) Z-spectra of EuL at $B_{1}=25.0 \mu \mathrm{T}$ and irradiation time $=5 \mathrm{~s}$; (bottom) determination of water exchange rates at $25^{\circ} \mathrm{C}$ (blue) and $37^{\circ} \mathrm{C}$ (red) at $B_{1}=35.0,30.0,25.0,22.5$, 20.0, 17.5, 15.0 and $10.0 \mu \mathrm{T}$, and irradiation time $=10 \mathrm{~s}$.

Table 1 Water exchange rates (in $\mathrm{Hz}$ ) obtained for EuL using the two independent methods

\begin{tabular}{llc}
\hline Temperature & Omega plots & QUEST/QUESP \\
\hline $25{ }^{\circ} \mathrm{C}$ & $1176 \pm 22$ & $930 \pm 11$ \\
$37^{\circ} \mathrm{C}$ & $2036 \pm 110$ & $2000 \pm 138$
\end{tabular}

protons (ESI $\dagger$ ), probably for the same reasons related to slow water exchange as in EuL. ${ }^{19,20}$

${ }^{19}$ F NMR spectra of GdL, EuL and TbL were recorded and ${ }^{19} \mathrm{~F}$ relaxation rates were determined to estimate the potential of these agents for ${ }^{19} \mathrm{~F}$ MRI. GdL displayed a single broad ${ }^{19} \mathrm{~F}$ NMR resonance, indicating substantial shortening of the relaxation times, while EuL and TbL revealed the existence of at least three paramagnetic species in the solution with different ${ }^{19} \mathrm{~F}$ chemical shifts (ESI $\dagger$ ). While the two peaks with higher intensities can be assigned to the common monocapped square-antiprismatic and monocapped twisted square-antiprismatic (SAP and TSAP, respectively) isomers of this type of compound, ${ }^{21}$ the appearance of the third resonance with very weak intensity can be explained by racemization of the starting amino acid or racemization of alkylating arms prior to or during alkylation of cyclen, as previously observed for similar systems under comparable experimental conditions. ${ }^{22}$ Consequently, ${ }^{19} \mathrm{~F}$ relaxation rates were determined only for the most abundant peaks (Table 2). 
Table $2{ }^{19} \mathrm{~F}$ relaxation rates of $\mathrm{GdL}$, EuL and $\mathrm{TbL}\left(300 \mathrm{MHz}, 25^{\circ} \mathrm{C}\right)$

\begin{tabular}{lcc}
\hline Temperature & ${ }^{19} \mathrm{~F} R_{1}(\mathrm{~Hz})$ & ${ }^{19} \mathrm{~F} R_{2}(\mathrm{~Hz})$ \\
\hline GdL & 182 & 385 \\
EuL & $1.4^{a, b}$ & $18^{a}$ \\
& $32^{a}$ & $19^{b}$ \\
TbL & $12^{b}$ & $48^{a}$ \\
& $28^{b}$ \\
${ }^{a}$ More abundant isomer. ${ }^{b}$ Less abundant isomer. & \\
\hline
\end{tabular}

As expected, GdL dramatically affected both ${ }^{19} \mathrm{~F} R_{1}$ and $R_{2}$, while still keeping a favorable $R_{1} / R_{2}$ ratio to obtain a good signal-tonoise ratio (SNR), when using ultrafast sequences. ${ }^{23}$ EuL and TbL also enhanced ${ }^{19} \mathrm{~F} R_{1}$ and $R_{2}$, however the reduction in the $R_{1} / R_{2}$ ratio (in the case of EuL) or larger signal splitting (in the case of $\mathbf{T b L}$ ) suggests GdL as a better candidate for ${ }^{19} \mathrm{~F}$ MRI studies, with the current DOTA-type chelator.

The potential of these systems to serve as multicontrast agents was demonstrated using in vitro MRI on tube phantoms (Fig. 3a-d). Four tubes containing GdL, EuL, TbL and water as controls were imaged using a 7T MRI scanner by different imaging protocols and frequencies. The greatest effect on relaxation times and hence the signal enhancement in $T_{1}$-weighted MRI experiments was observed for GdL (Fig. 3a), as would be expected; this effect was also confirmed by $T_{2}$-weighted MRI experiments (Fig. 3b). All three complexes displayed very good ${ }^{19} \mathrm{~F}$ MRI contrasts using the sequence parameters adjusted to assume different ${ }^{19} \mathrm{~F}$ relaxation rates due to influence of different paramagnetic ions (Fig. 3c). Lastly, only EuL exhibited a strong contrast in the CEST MRI experiment (Fig. 3d), as already indicated above in the NMR CEST experiments.

To confirm this platform as a multicontrast MRI agent in a complex environment, we have carried out an MRI study on animals using GdL and EuL. The contrast agent was injected intracranially into the somatosensory cortex of the anesthetized rats outside the scanner. For GdL, the animal was transferred into the scanner, and a very strong $T_{1}$-weighted MRI signal was recorded in vivo (Fig. 3e). Slow reduction in the MRI signal over a period of a few hours indicated very slow diffusion of GdL and its potential interaction with brain tissues. Although a similar behavior was previously observed with aminobisphosphonatecontaining contrast agents, ${ }^{24}$ it is hard to rationalize this effect with the current chelating platform since its diffusion properties in solution did not indicate any aggregation, i.e. the diffusion coefficient corresponded to other monomeric agents of similar size (ESI $\dagger)$.

Additionally, the effect of EuL was assessed by means of MRI ex vivo. A weak $T_{1}$-weigthed MRI contrast was obtained as expected after the animal was euthanized and transferred into the scanner (Fig. 3f). In parallel, a strong CEST contrast $(\sim 10 \%$ signal change) was successfully obtained at the frequency of the inner-sphere water molecule bound to $\mathrm{Eu}^{3+}$ (Fig. 3g), confirming the great potential of EuL for further paraCEST studies due to its long $\tau_{\mathrm{M}}$ (vide supra). In regard to ${ }^{19} \mathrm{~F} \mathrm{MRI}$, several imaging sequences were tested on both $\mathbf{G d L}$ and $\mathbf{E u L}$, using non-fluorine containing anesthesia to avoid possible interferences at the fluorine frequency. However, only a slight change in signal intensity could
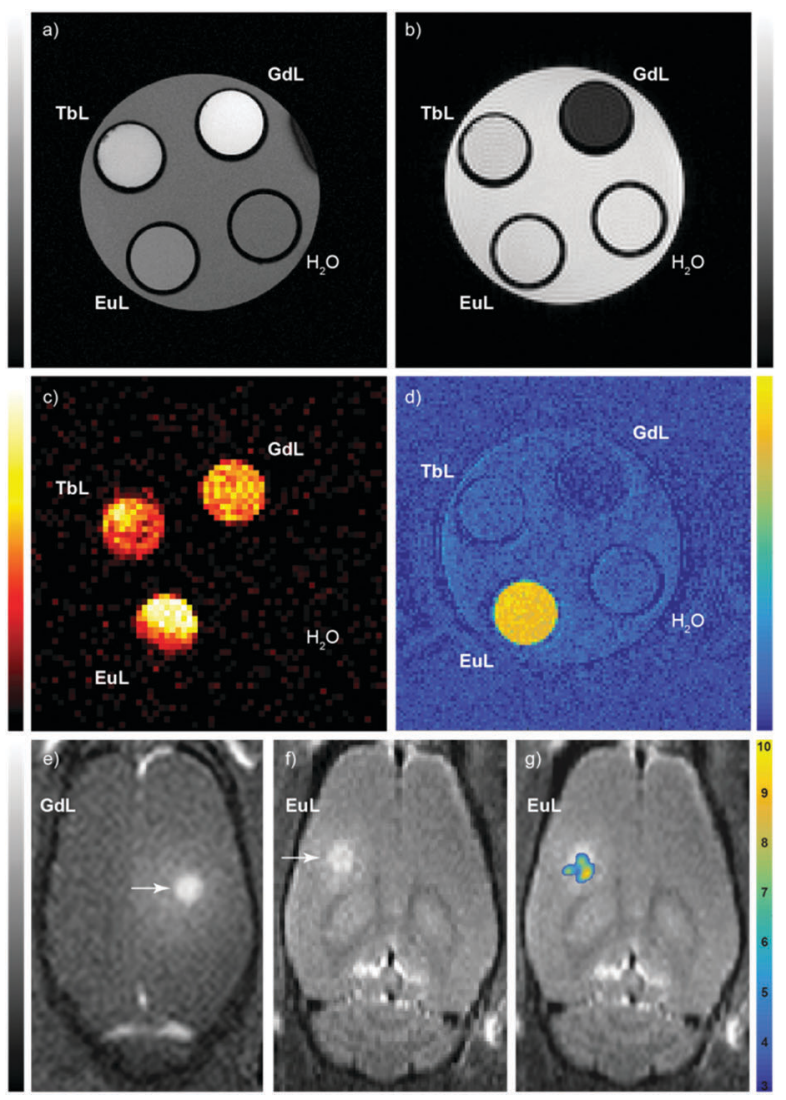

Fig. $3 \mathrm{MRI}$ experiments with the multicontrast agent. In vitro MRI on tube phantoms with $5 \mathrm{mM}$ of complexes (pH 7.3, HEPES, $25^{\circ} \mathrm{C}$ ): (a) $T_{1}$-weighted MRI; (b) $T_{2}$-weighted MRI; (c) ${ }^{19} \mathrm{~F} \mathrm{MRI;} \mathrm{(d)} \mathrm{paraCEST} \mathrm{MRI.} \mathrm{In} \mathrm{vivo} \mathrm{(GdL)} \mathrm{and}$ ex vivo (EuL) MRI in rat cortex: (e) $T_{1}$-weighted MRI with GdL; (f) $T_{1}$-weighted MRI with EuL; (g) merged CEST and $T_{1}$-weighted MRI with EuL. Arrows indicate the region where the contrast agent was injected; bars show signal intensity in arbitrary units except \%CEST in (g).

be detected after 2.3 hours of monitoring. As already discussed above, the lack of signals could indicate an interaction of the agent with surrounding tissues, which significantly reduces ${ }^{19} \mathrm{~F} T_{2}$ relaxation times and leads to signal disappearance (a notable broadening of the ${ }^{19} \mathrm{~F}$ signal in the measured $1 \mathrm{D}$ spectrum was observed for GdL). However, this effect can likely be avoided with another experimental design where the tissue density is lower (e.g. in the blood stream or kidneys), or by combining this platform to a nanosized system that will prevent any interaction with the tissue and improve the agent's biocompatibility. ${ }^{25}$

In conclusion, we report a promising platform for the development of multicontrast agents for MRI. A small size molecule accommodates different paramagnetic ions and subsequently enhances ${ }^{1} \mathrm{H} T_{1}$-weighted, ${ }^{1} \mathrm{H}$ CEST, and ${ }^{19} \mathrm{~F}$ MRI contrasts. The GdL and EuL complexes can concurrently serve as ${ }^{1} \mathrm{H} T_{1}$-weighted and ${ }^{19} \mathrm{~F}$ MRI, or as ${ }^{1} \mathrm{H}$ CEST and ${ }^{19} \mathrm{~F}$ MRI agents, respectively. As these two complexes are expected to have essentially the same biodistribution, the three different contrast mechanisms could be exploited using the same molecular platform, even if GdL and EuL have to be administered separately. ${ }^{26}$ Furthermore, the installation of the aromatic fluorinated units likely caused extremely advantageous exchange rates in EuL for CEST experiments, ${ }^{20}$ 
while possibly deteriorating the agent's biocompatibility for ${ }^{19}$ F MRI. Nevertheless, further improvements should easily be envisaged to provide the optimized multicontrast agent. Structural optimizations towards adjustment of the chelator's coordination properties may lead to a single isomer species that will be beneficial for ${ }^{19} \mathrm{~F}$ MRI. Synthetic modifications can lead to a higher number of fluorine atoms per molecule to increase the signal intensity, while a combination with various nanocarriers can improve the delivery, biokinetics and potentiality also the $T_{2}$ contrast of this multicontrast agent. The ability to collect different types of information from a single imaging probe just by using different imaging protocols brings new quality to MRI and can be a great asset for current molecular imaging to study various biological phenomena.

The authors thank Dr Kai Buckenmaier and Dr Dávid Balla for help in establishing appropriate anesthesia and MRI protocols and the Institute for Organic Chemistry, Faculty of Science, University of Tübingen, for the support in performing MS and elemental analyses. The financial support of the Max-Planck Society, the German Academic Exchange Service (DAAD, PhD fellowship to T.S.) and Ministerio de Economía y Competitividad (CTQ2013-43243-P and CTQ2015-71211-REDT, support to C. P.-I.) is gratefully acknowledged.

The animal experiments were approved by the local authorities (Regierungspraesidium), and were in compliance with the guidelines of the European directive (2010/63/EU) for the care and protection of animals used for scientific purposes.

\section{Notes and references}

1 R. Weissleder and M. J. Pittet, Nature, 2008, 452, 580-589.

2 H. Kobayashi, M. R. Longmire, M. Ogawa and P. L. Choyke, Chem. Soc. Rev., 2011, 40, 4626-4648.

3 S. R. Cherry, Annu. Rev. Biomed. Eng., 2006, 8, 35-62.

4 A. Y. Louie, Chem. Rev., 2010, 110, 3146-3195.

5 P. Caravan, in Molecular and cellular MR imaging, ed. M. M. J. Modo and J. W. M. Bulte, CRC Press, Taylor \& Francis Group, Boca Raton, London, New York, 2007, pp. 13-36.

6 A. D. Sherry and M. Woods, Annu. Rev. Biomed. Eng., 2008, 10, 391-411.
7 S. Aime, C. Carrera, D. D. Castelli, S. G. Crich and E. Terreno, Angew. Chem., Int. Ed., 2005, 44, 1813-1815.

8 S. Viswanathan, S. J. Ratnakar, K. N. Green, Z. Kovacs, L. M. De Leon-Rodriguez and A. D. Sherry, Angew. Chem., Int. Ed., 2009, 48, 9330-9333.

9 E. Terreno, D. D. Castelli, A. Viale and S. Aime, Chem. Rev., 2010, 110, 3019-3042.

10 I. Tirotta, V. Dichiarante, C. Pigliacelli, G. Cavallo, G. Terraneo, F. B. Bombelli, P. Metrangolo and G. Resnati, Chem. Rev., 2015, 115, 1106-1129.

11 M. Srinivas, A. Heerschap, E. T. Ahrens, C. G. Figdor and I. J. M. d. Vries, Trends Biotechnol., 2010, 28, 363-370.

12 S. Aime, A. Barge, D. Delli Castelli, F. Fedeli, A. Mortillaro, F. U. Nielsen and E. Terreno, Magn. Reson. Med., 2002, 47, 639-648.

13 E. Brücher, G. Tircsó, Z. Baranyai, Z. Kovács and A. D. Sherry, The Chemistry of Contrast Agents in Medical Magnetic Resonance Imaging, John Wiley \& Sons, Ltd, 2013, pp. 157-208.

14 P. Harvey, I. Kuprov and D. Parker, Eur. J. Inorg. Chem., 2012, 2015-2022.

15 S. Aime, A. Barge, J. I. Bruce, M. Botta, J. A. K. Howard, J. M. Moloney, D. Parker, A. S. de Sousa and M. Woods, J. Am. Chem. Soc., 1999, 121, 5762-5771.

16 W. T. Dixon, J. M. Ren, A. J. M. Lubag, J. Ratnakar, E. Vinogradov, I. Hancu, R. E. Lenkinski and A. D. Sherry, Magn. Reson. Med., 2010, 63, 625-632.

17 M. T. McMahon, A. A. Gilad, J. Y. Zhou, P. Z. Sun, J. W. M. Bulte and P. C. M. van Zijl, Magn. Reson. Med., 2006, 55, 836-847.

18 W. S. Fernando, A. F. Martins, P. Zhao, Y. Wu, G. E. Kiefer, C. PlatasIglesias and A. D. Sherry, Inorg. Chem., 2016, 55, 3007-3014.

19 X. J. Wang, Y. K. Wu, T. C. Soesbe, J. Yu, P. Y. Zhao, G. E. Kiefer and A. D. Sherry, Angew. Chem., Int. Ed., 2015, 54, 8662-8664.

20 A. D. Sherry and Y. K. Wu, Curr. Opin. Chem. Biol., 2013, 17, 167-174.

21 J. A. Peters, K. Djanashvili, C. F. G. C. Geraldes and C. Platas-iglesias, The Chemistry of Contrast Agents in Medical Magnetic Resonance Imaging, John Wiley \& Sons, Ltd, 2013, pp. 209-276.

22 T. Mani, A. C. L. Opina, P. Y. Zhao, O. M. Evbuomwan, N. Milburn, G. Tircso, C. Kumas and A. D. Sherry, JBIC, J. Biol. Inorg. Chem., 2014, 19, 161-171.

23 F. Schmid, C. Höltke, D. Parker and C. Faber, Magn. Reson. Med., 2013, 69, 1056-1062.

24 I. Mamedov, S. Canals, J. Henig, M. Beyerlein, Y. Murayama, H. A. Mayer, N. K. Logothetis and G. Angelovski, ACS Chem. Neurosci., 2010, 1, 819-828.

25 K. J. Thurecht, I. Blakey, H. Peng, O. Squires, S. Hsu, C. Alexander and A. K. Whittaker, J. Am. Chem. Soc., 2010, 132, 5336-5337.

26 A. M. Funk, V. Clavijo Jordan, A. D. Sherry, S. J. Ratnakar and Z. Kovacs, Angew. Chem., Int. Ed., 2016, 55, 5024-5027. 